\title{
Dynamic Lisfranc Joint Repair Concept: Surgical Technique for a Synthetic Neoligamentplasty
}

\author{
C. Nery ${ }^{1}$, E. Giza², E. Wagner ${ }^{3}$, M. A. Percope de Andrade ${ }^{4}$, T. Soares Baumfeld ${ }^{4}$, \\ M. Pires Prado 5 , D. Baumfeld ${ }^{4}$ \\ 1 Foot and Ankle Clinic, UNIFESP - Federal University of São Paulo, Brazil \\ 2 Foot \& Ankle Surgery Department of Orthopaedics, University of California, Davis \\ 3 Foot and Ankle Unit Clinica Alemana, Santiago, Chile \\ 4 Department of the Locomotor Apparatus, UFMG - Federal University of Minas Gerais, Belo Horizonte, MG, \\ Brazil \\ 5 Foot and Ankle Clinic, Albert Einstein Hospital, São Paulo, SP, Brazil
}

\section{CORRESPONDING AUTHOR:}

\section{Daniel Baumfeld}

Rua engenheiro albert sharle 30

30380370 Luxemburgo, MG, Brazil

E-mail: danielbaumfeld@gmail.com

DOI:

10.32098/mltj.04.2019.11

LEVEL OF EVIDENCE: 5

\begin{abstract}
SUMMARY
Background. Isolated Lisfranc ligament and some other combined ligament injuries to the Lisfranc Joint Complex are frequently found in clinical practice, and surgical treatment is commonly indicated. In order to achieve favorable outcomes following the repair of Lisfranc injuries, a successful anatomical reduction is required. Various fixation methods currently exist such as screw fixation, dorsal bridge plate fixation, K-wire stabilization, primary fusion, neoligamentplasty and TightRope stabilization. Each technique has its own advantages and disadvantages. any rigid and long immobilization of a ligament is detrimental to healing and compromises its mechanical properties. Ideally, the best form to treat an articular joint involvement is to use a long-lasting implant, with flexibility that could potentially provide the proper stability allowing enough motion to achieve a more physiologic ligament healing. We describe a novel technique to reconstruct the Lisfranc injuries with pure ligament disruption using a synthetic neoligamentplasty with FiberTape - InternalBrace ${ }^{\mathrm{TM}}$ (Arthrex, Naples, FL) passed through anatomic bone tunnels that mimic the original isometric position of the ligaments.

Methods. We described the technique and evaluated 7 consecutive patients with midfoot ligament injury and a combination of tarsometatarsal instability and/or intercuneiform instability.

Results. We found no postoperative complications, stiffness, or loss of correction.

Conclusions. The innovative surgical technique present in this article is effective and eliminates the need for subsequent implant removal for lisfranc joint injuries. It could also provide a more physiologic arch deformation to interact with the environment in a more effective way, protecting the surrounding joints from excessive loads.
\end{abstract}

\section{KEY WORDS}

lisfranc joint; neoligamentplasty; subtle Lisfranc

\section{INTRODUCTION}

Isolated Lisfranc ligament and some other combined ligament injuries to the Lisfranc Joint Complex are frequently found in clinical practice, and surgical treatment is commonly indicated (1). Because of the close relationship of the tarsometatarsal bones and the relatively restricted mobility of each one of the joints in this area, it is intuitive to conclude that isolated Lisfranc ligament lesions are very rare to say the less (2). Considering the Nunley-Vertullo's classification for the tarsometatarsal ligament lesions, grades 
II and III show important characteristics that can lead to regional instability, because of the diastasis between the first and second metatarsals and the intercuneiform joints (3).

Anatomic reduction of the Lisfranc joint following an injury is demanding. Outcomes are direct correlated with it. As a concept used for other joints, stable fixation of an articular injury provides the best results. Fixation of the medial, lateral and intermediate cuneiforms with trans-articular screws provide a stable fixation, but it destroys part of the articular surface and there is a necessity of implant removal to try to reestablish the articular function (4-7).

Primary arthrodesis is an option reported in the literature to treat the tarsometatarsal injuries $(2,8)$. Arthrodesis eliminate normal motion of theses joints and may overload the adjacent joint. In a recent study, it was proved that patients who were treated with arthrodesis or plate/screw fixation had significantly lower walking speed and significantly decreased flexion/extension in the midfoot during the push-off phase (9). This is a functional demonstration of the limitations that could occur after this type of treatment. Nowadays, the literature still consider the use of trans-articular screws or primary arthrodesis to treat Lisfranc injuries as gold standard, $(1,2,10)$ even with the common concept that any rigid and long immobilization of a ligament is detrimental to healing and compromises its mechanical properties (11). Ideally, the best form to treat an articular joint involvement is to use a long-lasting implant, with flexibility that could potentially provide the proper stability allowing enough motion to achieve a more physiologic ligament healing.

There are two options in the literature for non-rigid Lisfranc stabilization: Neoligamentplasty with allograft/autograft or tightrope fixation $(12,13)$.
A biomechanical publication analyzing the differences between screw fixation and Mini TightRope ${ }^{\circledR}$ (Arthrex, Naples, FL) for Lisfranc injury shows that the strength of the Mini TightRope ${ }^{\circledR}$ is not much different than a $3.5-\mathrm{mm}$ or a 4.5 -mm screw $(14,15)$.

In the same way, neoligamentplasty has been demonstrated to be a safe option for acute and late reconstruction of Lisfranc ligament injury (11-13).

We describe a innovative technique to reconstruct the Lisfranc injuries with pure ligament disruption using a synthetic neoligamentplasty with FiberTape - InternalBrace $^{\mathrm{TM}}$ (Arthrex, Naples, FL) passed through anatomic bone tunnels that mimic the original isometric position of the ligaments and a retrospective analysis of the first patients submitted to this technique.

\section{METHODS}

\section{Patients}

Seven patients were retrospectively evaluated and included into this study. All of them had a history of an acute midfoot ligament injury and a combination of tarsometatarsal instability and/or intercuneiform instability. Mean age was 36 years $(\min =15 / \max =55$ years $)$. There were 4 males and 3 females (table I). The inclusion criteria were: (1) adult patients with history of an acute closed injury of the tarsometatarsal joint; (2) Physical examination suggestive and compatible with tarso-metatarsal and/or tarsometatarsal acute instability (pain and swelling on the dorsal aspect of the midfoot; intense pain and some degree of instability during pronation-supination manual test; and plantar ecchymosis) (3) Radiological signals of Lisfranc ligament

Table I. Demographic Data

\begin{tabular}{llllll}
\hline$\#$ & Gender & Age (years) & Side & Pattern of Ligaments Involvement & Trauma Agent \\
\hline 1 & $\mathrm{~F}$ & 44 & $\mathrm{R}$ & $(\mathrm{C} 1-\mathrm{C} 2)+(\mathrm{C} 1-\mathrm{Mt} 2)+(\mathrm{C} 1-\mathrm{Mt} 1)+(\mathrm{C} 2-\mathrm{Mt} 2)$ & Fall on the stairs \\
\hline 3 & $\mathrm{M}$ & 29 & $\mathrm{R}$ & $\begin{array}{l}(\mathrm{C} 1-\mathrm{C} 2)+(\mathrm{C} 1-\mathrm{Mt} 2)+(\mathrm{C} 1-\mathrm{Mt} 1)+(\mathrm{C} 2-\mathrm{Mt} 2)+(\mathrm{C} 2-\mathrm{C} 3) \\
+(\mathrm{C} 3-\mathrm{Mt} 3)\end{array}$ & Wakeboard \\
\hline 4 & $\mathrm{~F}$ & 37 & $\mathrm{~L}$ & $(\mathrm{C} 1-\mathrm{C} 2)+(\mathrm{C} 1-\mathrm{Mt} 2)+(\mathrm{C} 1-\mathrm{Mt} 1)+(\mathrm{C} 2-\mathrm{Mt} 2)$ & Windsurf \\
\hline 5 & $\mathrm{M}$ & 55 & $\mathrm{~L}$ & $(\mathrm{C} 1-\mathrm{C} 2)+(\mathrm{C} 1-\mathrm{Mt} 2)+(\mathrm{C} 2-\mathrm{Mt} 2)$ & Fall on the stairs \\
\hline 6 & $\mathrm{M}$ & 31 & $\mathrm{~L}$ & $(\mathrm{C} 1-\mathrm{C} 2)+(\mathrm{C} 1-\mathrm{Mt} 2)+(\mathrm{C} 2-\mathrm{Mt} 2)$ & Skateboard \\
\hline 7 & $\mathrm{M}$ & 36 & $\mathrm{R}$ & $(\mathrm{C} 1-\mathrm{C} 2)+(\mathrm{C} 1-\mathrm{Mt} 2)+(\mathrm{C} 1-\mathrm{Mt} 1)+(\mathrm{C} 2-\mathrm{Mt} 2)$ & Soccer \\
\hline $\mathrm{C} 1-$ & & & $(\mathrm{C} 1-\mathrm{C} 2)+(\mathrm{C} 1-\mathrm{Mt} 2)+(\mathrm{C} 2-\mathrm{Mt} 2)$ & foot sprain \\
\hline
\end{tabular}

C1-C2 - First Intercuneiform ligaments

C1-Mt2 - Lisfranc Ligament

C1-Mt1 - First Cuneometatarsal Ligament

C2-Mt2 - Second Cuneometatarsal Ligament

$\mathrm{C} 2-\mathrm{C} 3$ - Intercuneiform ligaments between $\mathrm{C} 2$ and C3

C3 - Mt3 - Third Cuneometatarsal Ligament 


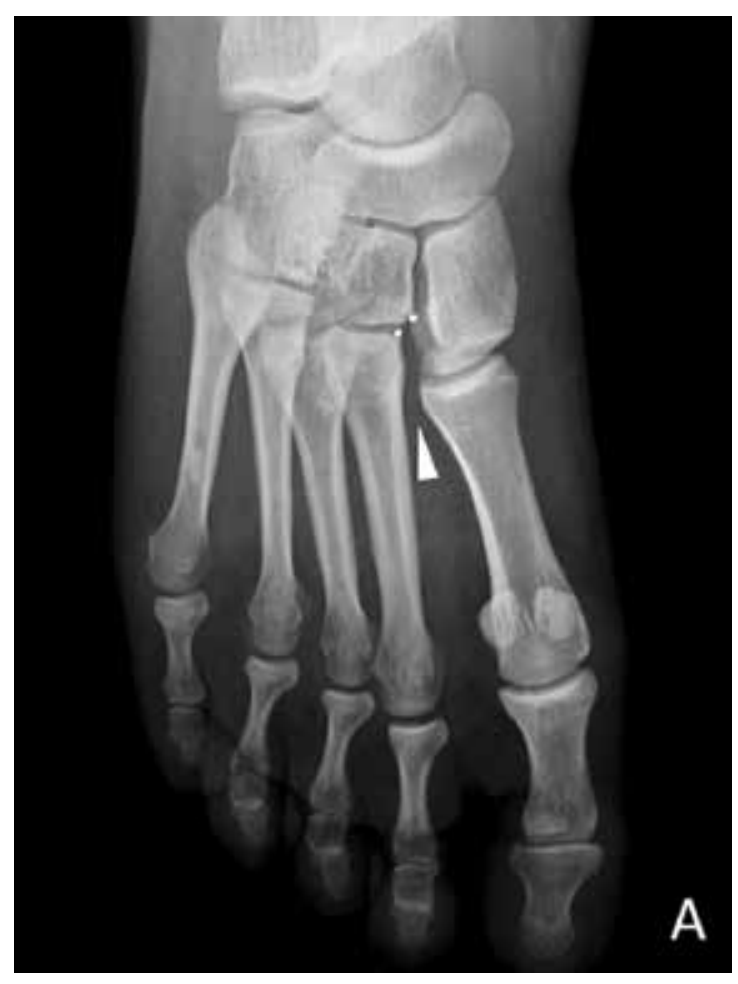

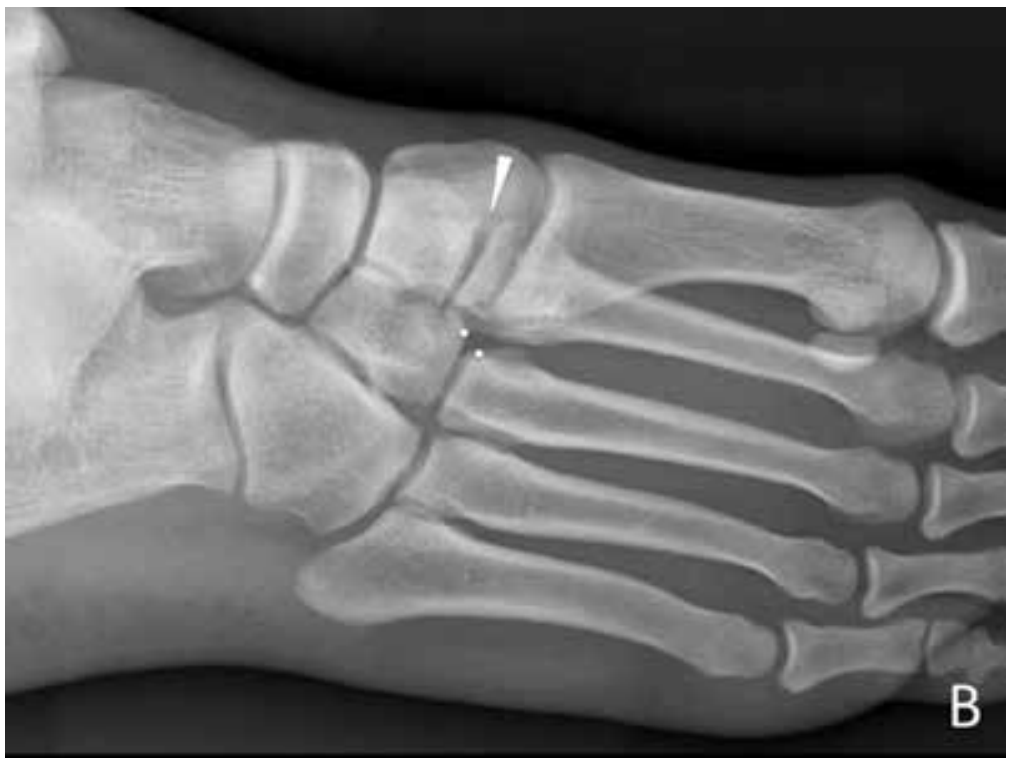

Figure 1. Tarsometatarsal pure ligament injury: A. widening of the spaces between the first and second cuneiforms and the bases of the first and second metatarsals (arrow head); B. Oblique view with asymmetry of the second tarsal-metatarsal joint (arrow head) and third cuneiform and third metatarsal bone (dots). injury and/or intercuneiform instability, represented by the widening of the medial cuneiform and the second metatarsal space, the first metatarsal and medial cuneiform deviation and the C1-C2 intercuneiform space widening (figure 1). Magnetic Resonance (MR) images were used to confirm the clinical and radiological findings by means of the identification of direct and indirect signals of Lisfranc and intercuneiform ligament disruption (figure 2). The Nunley and Vertulo Grading System were used to graduate and classify the injury (3).

Three patterns of lesions were found in our sample: (1) rupture of the First Intercuneiform ligament (C1-C2), Lisfranc ligament (C1-Mt2) and the Second Tarsometatarsal ligament (C2-Mt2); (2) Rupture of the First Intercuneiform ligament (C1-C2), Lisfranc ligament (C1-Mt2), Second Tarsometatarsal ligament (C2-Mt2) and the First Tarsometatarsal ligaments (C1-Mt1); and (3) First Intercuneiform ligament (C1-C2), Lisfranc ligament (C1-Mt2), First Tarsometatarsal ligaments (C1-Mt1), Second Tarsometatarsal ligaments (C2-Mt2), Second Intercuneiform ligaments (C2-C3) and Third Tarsometatarsal ligaments (C3-Mt3) (figure 3).

\section{Surgical Technique}

The patient is positioned in supine position. A thigh tourniquet is applied to provide a bloodless surgical field.
A $5-\mathrm{cm}$ linear incision located dorsally over the first intermetatarsal space give us access to the medial intertarsal and tarsometatarsal joints. With a careful evaluation of all anatomic structures of this region one can identify the involved ligaments and quantify the regional instability observing the joints behavior during pronation and supination of the forefoot. All the remain ligaments must be removed from every affected joint and interspaces in order to reestablish the normal relationship of the joints.

Accessory incisions may be necessary depending of the instability encountered. They can be made parallel to the first incision keeping a secure distance between them.

Once the joint instability has been confirmed and the injury pattern established, the second metatarsal base was anatomically repositioned using a bone clamp. Satisfactory reduction of all involved joints was confirmed with fluoroscopic images. Bone tunnels were strategically placed to allow the recreation of the main ligaments involved, restoring joint stability. Figure 4A shows the most important information regarding the bone tunnels preparation and placement. Tunnel \#1 starts at the medial border of the medial cuneiform and is directed to the base of the second metatarsal bone, reproducing the trajectory of the Lisfranc ligament. Tunnel \#2 begins at the medial border of the medial cuneiform, 3 or $4 \mathrm{~mm}$ proximal to the entrance of the tunnel \#1 and heads to the lateral dorsal region of the middle cunei- 


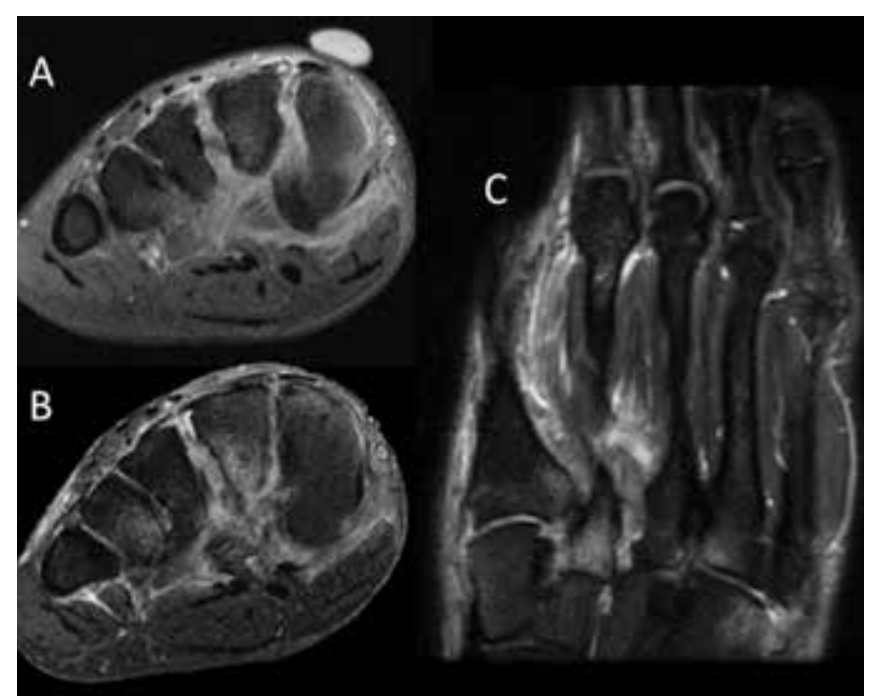

Figure 2. MRI images of pure Lisfranc ligamentous injury: A. T1 Axial Fat Gd; B. T2 Axial Fat; C. T2 Coronal Fat. The hyper signal observed in the spaces between the medial, intermediate and lateral cuneiforms. Coronal view (C) showing the absence of the Lisfranc.

form. Tunnel \#3 is performed from medial to lateral, in the coronal plane, $1 \mathrm{~cm}$ distal to the base of the first metatarsal. Tunnel \# 4 crosses obliquely the base of the third metatarsal bone aligning with the lateral hole of tunnel \#1. Finally, tunnel \#5 follows the same path of tunnel \#2, extending to the lateral cuneiform. Tunnels \#2 and \#5 are never used in the same patient, they will be chosen depending of the amount of instability encountered.

The bone tunnels needed for the simplest pattern of injury - $(\mathrm{C} 1-\mathrm{C} 2)+(\mathrm{C} 1-\mathrm{Mt} 2)+(\mathrm{C} 2-\mathrm{Mt} 2)-$ were tunnels \#1 and \#2. To address the intermediate complexity pattern - (C1-C2) $+(\mathrm{C} 1-\mathrm{Mt} 2)+(\mathrm{C} 1-\mathrm{Mt} 1)+(\mathrm{C} 2-\mathrm{Mt} 2)-$ it is necessary to perform tunnels \#1, \#2 and \#3. When it comes to the most complex lesion pattern $-(\mathrm{C} 1-\mathrm{C} 2)+(\mathrm{C} 1-\mathrm{Mt} 2)+(\mathrm{C} 1-\mathrm{Mt} 1)+$ $(\mathrm{C} 2-\mathrm{Mt} 2)+(\mathrm{C} 2-\mathrm{C} 3)+(\mathrm{C} 3-\mathrm{Mt} 3)-$ it is necessary to use four bone tunnels: tunnel \#1, \#5 instead of \#2, \#3 and\#4.

It is recommended to use $1.2 \mathrm{~K}$-wires to find the correct position of the tunnels under fluoroscopic guidance. Once this is achieved, the bone tunnels can be drilled using a 2.5 mm cannulated drill bit.

Using a litinol suture-passer, both ends of a \#2 FiberTape - InternalBrace ${ }^{\mathrm{TM}}$ (Arthrex, Naples, FL) are passed from medial to lateral through the tunnel \#1, leaving a lace exteriorized at the medial entrance of the tunnel. This step is the same for all injury patterns found in our series.

In the simplest and in the intermediate complexity patterns, both free ends of the tape are now carried over the second

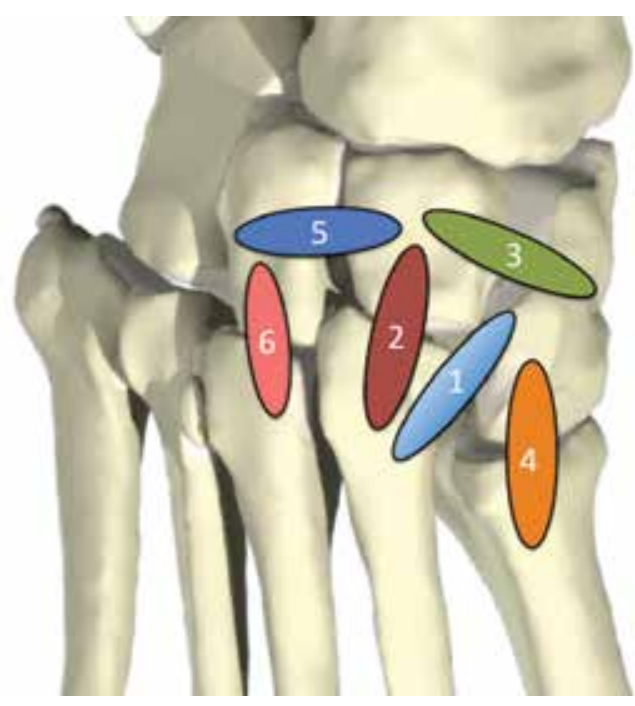

Figure 3. Injury Patterns: $1+2+3=$ Lisfranc +2 nd tarsal-metatarsal + First intercuneiform(C1-C2); $1+2+3+4=$ Lisfranc + 2nd tarsal metatarsal + First intercuneiform (C1-C2) + 1st tarsal-metatarsal; $1+2+3+4+5+6=$ Lisfranc +2 nd tarsal metatarsal + First intercuneiform (C1-C2) + 1st tarsal-metatar$\mathrm{sal}+$ Second intercuneiform (C2-C3) + 3rd tarsal-metatarsal.

tarsal-metatarsal joint and passed from lateral to medial through tunnel \#2.

Both ends of the tape are passed through the lace in order to allow adequate tensioning. One assistant kept the tension over the ends of the tape while the surgeon introduced two $3 \times 8 \mathrm{~mm}$ Bio-Tenodesis ${ }^{\mathrm{TM}}$ screws (Arthrex, Naples, FL) in the medial holes of both \#1 and \#2 tunnels, stabilizing the intercuneiform and tarsometatarsal joints. For the simplest pattern of injury - (C1-C2) + (C1-Mt2) + (C2-Mt2) - the above description addresses all the unstable joints and reconstructs all the injured ligaments, allowing both free ends of the tape to be cut (figure4B).

In the intermediate complexity pattern - (C1-C2) + (C1-Mt2) $+(\mathrm{C} 1-\mathrm{Mt} 1)+(\mathrm{C} 2-\mathrm{Mt} 2)-$ both ends of the tape were carried over the first tarsometatarsal joint and passed through bone tunnel \#3. One end of the tape is passed from medial to lateral and the other from lateral to medial. Tension is applied in both tape ends and a third $3 \times 8 \mathrm{~mm}$ Bio-Tenodesis $^{\mathrm{TM}}$ screw is introduced in the tunnel, from medial to lateral, to stabilize the construct (figure $4 \mathrm{C}$ ).

When dealing with the most complex lesion pattern $(\mathrm{C} 1-\mathrm{C} 2)+(\mathrm{C} 1-\mathrm{Mt} 2)+(\mathrm{C} 1-\mathrm{Mt} 1)+(\mathrm{C} 2-\mathrm{Mt} 2)+(\mathrm{C} 2-\mathrm{C} 3)+$ (C3-Mt3) -, just after passing the tape through the tunnel \#1, both free ends of the tape were passed through the tunnel \#4 at the base of the 3rd metatarsal, carried over the 3rd tarsal-metatarsal joint and passed through the bone tunnel \#5 in order to stabilize the injured ligaments. The fixation 
$4 \mathrm{~A}$

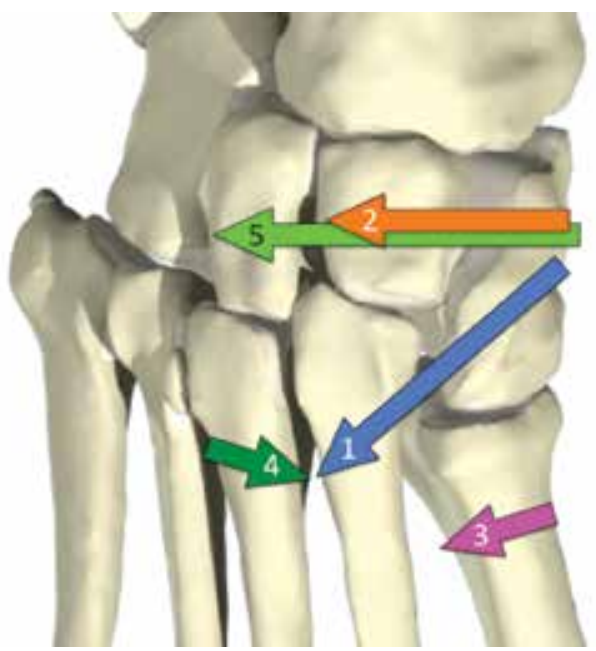

$4 \mathrm{C}$

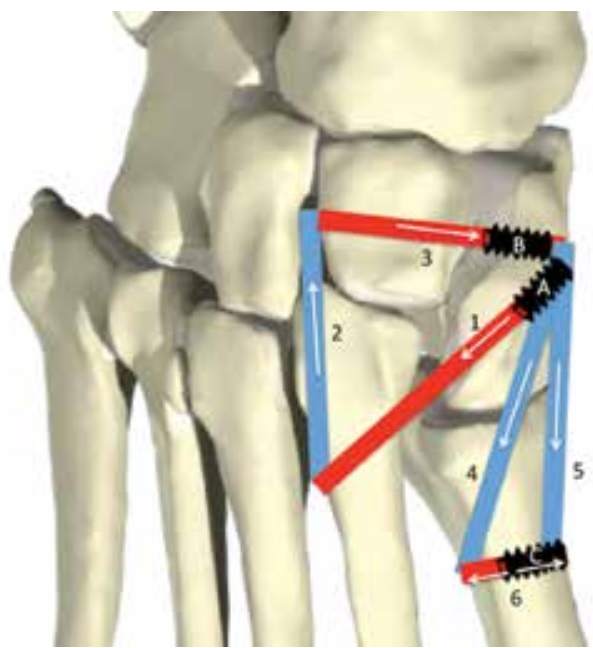

4B

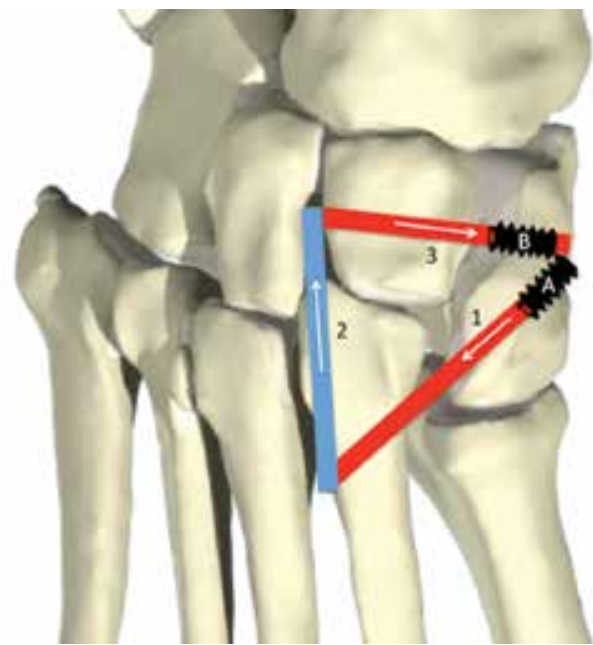

4D

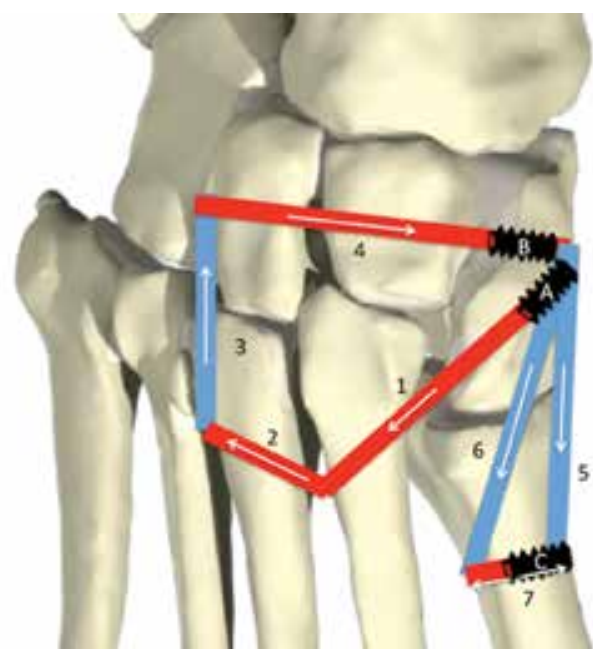

Figure 4A. positioning and length of the bone tunnels; B, C and D - Red lines indicate the bone tunnels; blue lines indicate the segments of superficial position of the FiberTape; the white arrows indicate the direction of introduction of the FiberTape while the numbers indicate the sequence used to pass the FiberTape in each bone tunnel or superficial way. The Bio-Tenodesis screws appear in black and the letters on them indicate the sequence of screw introduction.

$\mathrm{B}=(\mathrm{C} 1-\mathrm{C} 2)+(\mathrm{C} 1-\mathrm{Mt} 2)+(\mathrm{C} 2-\mathrm{Mt} 2)$ the simplest injury pattern; $\mathrm{C}=(\mathrm{C} 1-\mathrm{C} 2)+(\mathrm{C} 1-\mathrm{Mt} 2)+(\mathrm{C} 1-\mathrm{Mt} 1)+(\mathrm{C} 2-\mathrm{Mt} 2)$ the intermediate complexity injury pattern; $\mathrm{D}=-(\mathrm{C} 1-\mathrm{C} 2)+(\mathrm{C} 1-\mathrm{Mt} 2)+(\mathrm{C} 1-\mathrm{Mt} 1)+(\mathrm{C} 2-\mathrm{Mt} 2)+(\mathrm{C} 2-\mathrm{C} 3)+(\mathrm{C} 3-\mathrm{Mt} 3)$ represents the more complex injury pattern.

with screws and the stabilization of the first tarsometatarsal joint follows the same principles already described.

In figures $4 \mathrm{~B}$ to $4 \mathrm{D}$, one can find the detailed schemes of all the steps mentioned above and in figure $\mathbf{5}$, all the related surgical images.

At the end of the reconstruction procedure, stability and joint reduction were analyzed under direct and fluoroscopic visualization, including manual stress tests.
The postoperative protocol included 2 weeks in a short leg splint until sutures were removed and then transitioned to a non-weight-bearing boot for an additional 4 weeks. Physiotherapy program started at the 3 rd week without foot load. At the end of the sixth week, patients were allowed to weight-bear with boots as tolerated for two more weeks. Return to sports is permitted after 6 months.

All cases of Lisfranc injuries in our series were surgically treated by the same team. 

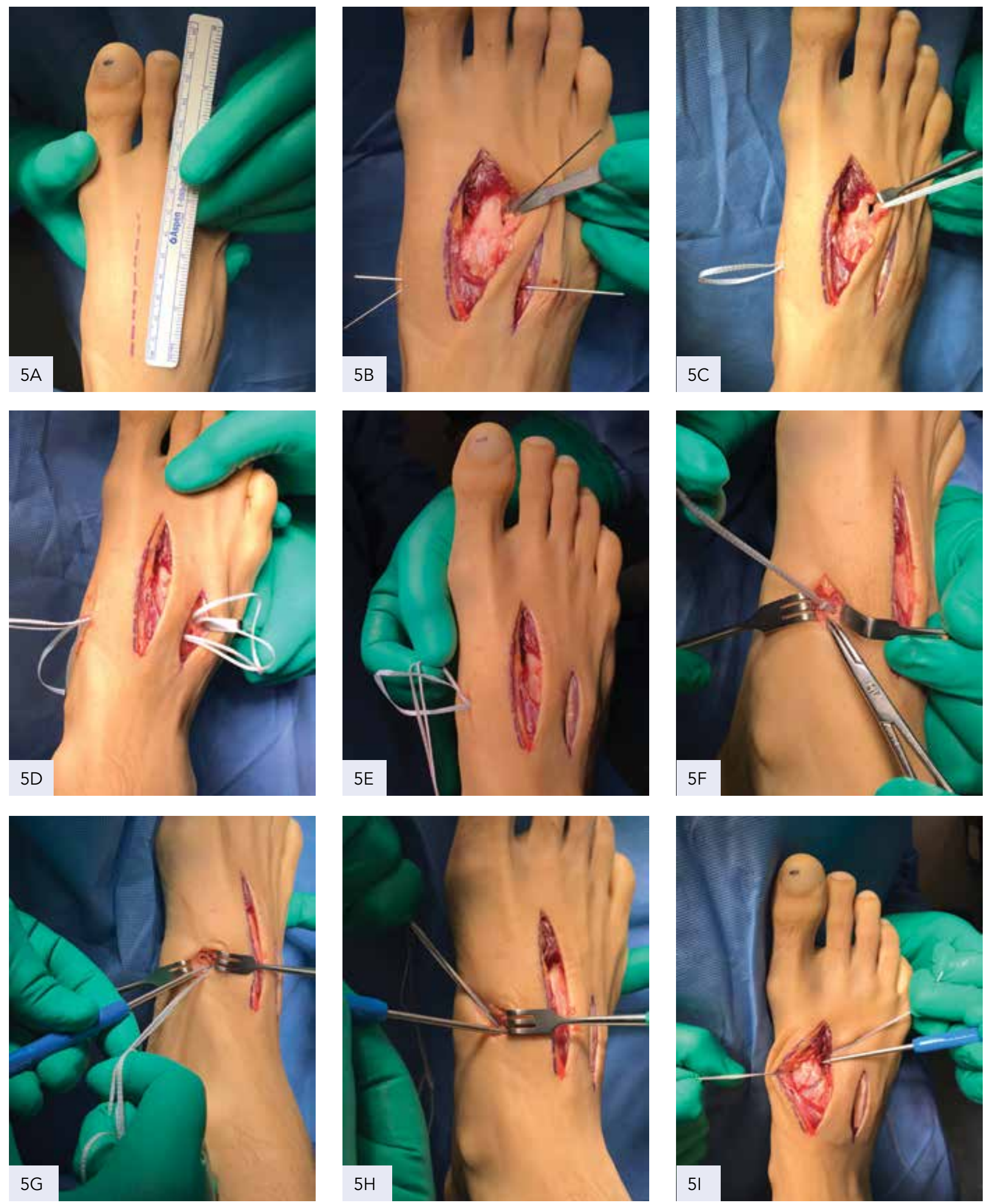

Muscles, Ligaments and Tendons Journal 2019;9 (4) 


\section{RESULTS}

All patients had radiographic and clinical improvement. Reduction of the tarsometatarsal, intercuneiform joint and restoration of the foot arch height was observed on weightbearing radiographs in all cases. (figure 6).

Every patient returned to normal activities within the observation period, i.e. 3 to 10 months.

The mean postoperative AOFAS score ranged from 89 to 98 points (table II). There were no postoperative wound complications or infections, construction failure, loss of reduction or post-traumatic arthritis.

\section{DISCUSSION}

Despite the fact of Lisfranc injuries are not common, they can be a disastrous injuries to normal population or in professional elite player(16). Different fixation methods exist such as screw fixation, dorsal bridge plate fixation, K-wire stabilization, primary fusion, neoligamentplasty and TightRope stabilization. Each technique has its own advantages and disadvantages (6).

Early reports of Lisfranc injury management using Kirschner wire fixation to maintain a stable reduction endorse this method, but the recent literature do not advocate it for the athletic population. Numerous studies have demonstrated improved stability with screw and/or plate fixation, which also limits the risks of recurrent subluxation after early pin removal and pin site complications (17).

The use of trans articular screw are most common method used to treat acute Lisfranc ligamentous injuries with good results reported. They provide adequate stability but they
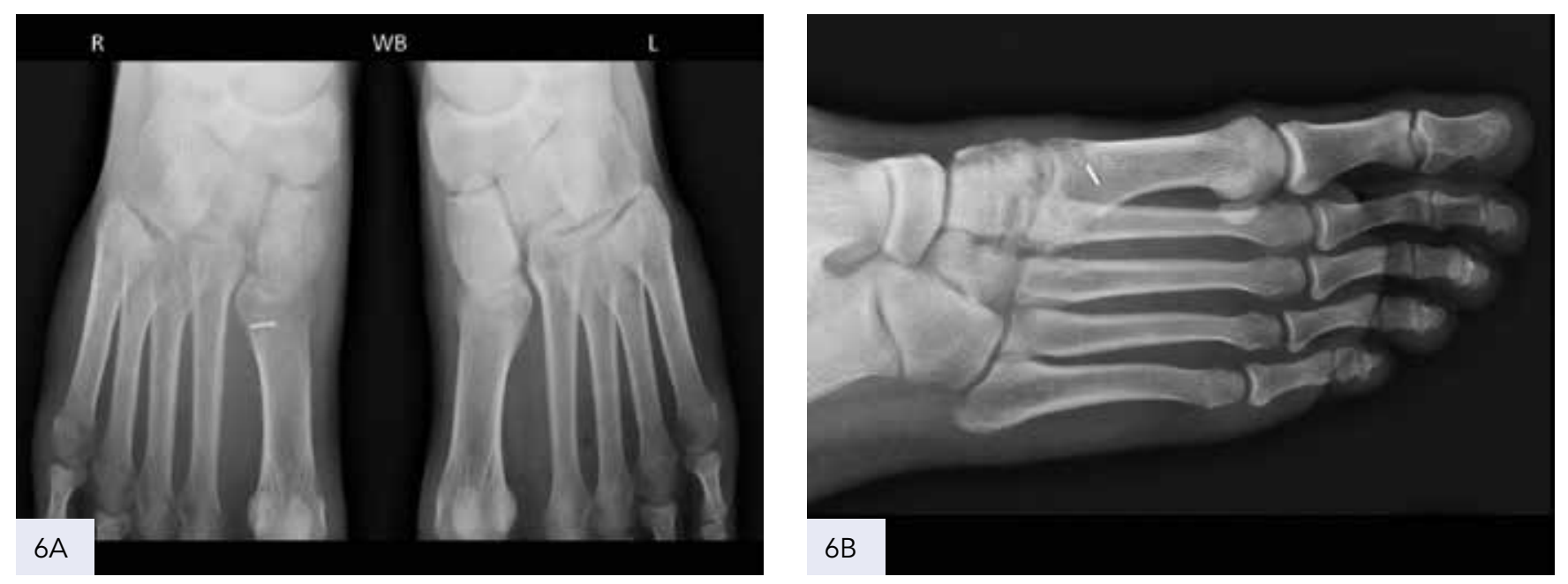

Figure 6 A-B. Radiograph presenting the normal restoration of the foot arch height and reduction of the tarsometatarsal, intercuneiform joint.

compromise the TMT joint articular surface and make the joint rigid and less adaptable in a stand position. $(4,17,18)$

The concept of midfoot ligament reconstruction and motion preservation breaks the common sense of immobilization to hope for ligament healing. The use of trans articular screws or a primary arthrodesis has never been considered in knee or shoulder ligament injuries, and, in that way, why should they be considered in a joint with movement and with an important biomechanical property in the foot? After Lisfranc injuries, patients have altered gait compared with healthy subjects (9). When gait analysis is performed in patients who have had a Lisfranc stabilization with trans articular screws or arthrodesis, they show a significantly lower walking speed and significantly decreased flexion/ extension in the midfoot during push-off phase. Patients with a rigid midfoot after arthrodesis or screw/plates fixation also present an increased plantar pressure in the late instants of stance phase, possibly due to arch stiffness changes (19). In addition, ROM has been significantly correlated with functional score but not with the radiographic reduction. This could indicate that in the post- operative phase a high flexion-extension motion is important for patient satisfaction. Even in patients where hardware was removed before gait analysis, they still presented a non-functional gait $(9,20)$. This last fact highlights the need of finding a reliable, functional and non-rigid reconstruction of midfoot injuries. The non-rigid method of fixation for midfoot ligament reconstruction either with autologous tissue or synthetic materials has already been described. Few reports exist, as the neoligamentoplasty for Lisfranc joint instability. Different authors had demonstrated the use of different autografts 
Table II. Pöst operative data

\begin{tabular}{cccc}
\hline$\#$ & Nunley-Vertullo & Follow-up & AOFAS Post-op \\
\hline 1 & III & 10 & 95 \\
\hline 2 & III & 7 & 90 \\
\hline 3 & III & 6 & 98 \\
\hline 4 & II & 5 & 92 \\
\hline 5 & II & 5 & 90 \\
\hline 6 & III & 4 & 92 \\
\hline 7 & II & 3 & 89 \\
\hline mean & & 6 & 92 \\
\hline
\end{tabular}

(third extensor digitorum longus, gracilis, half of the extensor hallucis longus tendon) to reconstruct midfoot ligament injuries, with good results in acute and chronic injuries even in professional athletes $(5,12,13,21,22)$. Precise placement of bone tunnels to guide the grafts has been described too, following the anatomic position of the ligaments, which should be paramount to achieve a correct reconstruction (13). Alternative materials such as synthetic sutures have been suggested too, such as the method described using the Tightrope (Arthrex) technique, which avoids donor site morbidity. Recent studies have confirmed equivalent stability across the Lisfranc joint when using a suture button device as compared with screw fixation.(20)

In this article, we have provided a technique to treat acute tarsometatarsal and/or intercuneiform ligamentous injuries in selected cases. This technique has some advantages compared with other non-rigid fixation alternatives. It allows fixation of all types of injuries with multidirectional ligamentous disruption, avoiding donor site morbidity. It maintains joint motion which could also provide a more physiologic gait. It also avoids screw breakage and the need for implant removal $(16,23,24)$.

This study presents some limitations. it is a level V case series of a very small number of patients with no control group, the results of the different types of surgical procedures, such as screw fixation or primary arthrodesis, could not be estimate. We also need a biomechanical study to directly compare rigid versus non rigid fixation techniques for midfoot injuries.

The potential complication that could exist should also be advised. The tunnels must follow the correct description to mimic the normal ligament, their incorrect position could lead to cartilage and joint destruction and future arthrosis. Also, to tight the synthetic tape, surgeons need a good bone structure, its use in a osteoporotic bone or if the tunnels were not performed in the proper position a bone fail could occur.
In summary, we present in this article a new technique used to stabilize Lisfranc fracture dislocations. This method is effective and eliminates the need for subsequent implant removal. As the repair follows the ligament trajectories and insertions, it may possibly simulate the mechanical properties of the ligaments until they heal. Furthermore, diastasis should not be a concern because very strong, nonabsorbable tapes are used to restore the strength and integrity of the joints until the TMT ligaments heal. We believe a non-rigid fixation enhances healing, preserves joint motions and provides equivalent results compared with screw fixation. This type of fixation could also provide a more physiologic arch deformation to interact with the environment in a more effective way, protecting the surrounding joints from excessive loads. A prospective research is needed before the present technique can become the standard operative treatment of ligamentous tarso-metatarsal injuries.

\section{CONCLUSIONS}

The surgical technique present in this article is effective and dispense the necessity of implant removal for Lisfranc joint injuries. It could also provide a more physiologic arch deformation to connect with the environment in a more efficient way, protecting the surrounding joints from overloads.

\section{CONFLICT OF INTERESTS}

The authors declare they have no conflict of interests

\section{REFERENCES}

1. Welck MJ, Zinchenko R, Rudge B. Lisfranc injuries. Injury 2015;46:536-41. doi:10.1016/j.injury.2014.11.026.

2. Seybold JD, Coetzee JC. Lisfranc Injuries. Clinics in Sports Medicine 2015;34:705-23. doi:10.1016/j.csm.2015.06.006.

3. Nunley JA, Vertullo CJ. Classification, Investigation, and Management of Midfoot Sprains. The American Journal of Sports Medicine 2002;30:871-8.

4. Lau S, Howells N, Millar M, De Villiers D, Joseph S, Oppy A. Plates, Screws, or Combination? Radiologic Outcomes After Lisfranc Fracture Dislocation. The Journal of Foot and Ankle Surgery 2016;55:799-802. doi:10.1053/j.jfas.2016.03.002.

5. Romero J. Classification and management of Lisfranc joint injuries: current concepts. Current Orthopaedic Practice 2016;27:1-6.

6. Desmond EA, Chou LB. Current Concepts Review: Lisfranc Injuries. Foot Ankle Int 2016;27:653-60. doi:10.1177/ 107110070602700819.

7. Lau S, Guest C, Hall M, Tacey M, Joseph S, Oppy A. Functional Outcomes Post Lisfranc Injury-Transarticular Screws, Dorsal Bridge Plating or Combination Treatment? Journal of Orthopaedic Trauma 2017;31:447-52. doi:10.1097/ BOT.0000000000000848. 
8. Qiao Y-S, Li J-K, Shen H, Bao H-Y, Jiang M, Liu Y, et al. Comparison of Arthrodesis and Non-fusion to Treat Lisfranc Injuries. Orthop Surg 2017;9:62-8. doi:10.1111/os.12316.

9. van Hoeve S, Stollenwerck G, Willems P, Witlox MA, Meijer K, Poeze M. Gait analysis and functional outcome in patients after Lisfranc injury treatment. Foot and Ankle Surgery 2017:1-7. doi:10.1016/j.fas.2017.07.003.

10. Krause F, Schmid T, Weber M. Current Swiss Techniques in Management of Lisfranc Injuries of the Foot. Fcl 2016;21:33550. doi:10.1016/j.fcl.2016.01.006.

11. Weglein DG, Andersen CR, Morris RP, Buford WL Jr, Panchbhavi VK. Allograft Reconstruction of the Lisfranc Ligament. Foot \& Ankle Specialist 2015;8:292-6. doi:10.1177/1938640015584768.

12. Nery C, Raduan F, Baumfeld D. Joint-sparing Corrections in Malunited Lisfranc Joint Injuries. Foot Ankle Clin N Am 2016;21:161-76. doi:10.1016/j.fcl.2015.09.007.

13. Nery C, Réssio C, Alloza JFM. Subtle Lisfranc Joint Ligament Lesions:. Foot and Ankle Clinics of NA 2012;17:407-16. doi:10.1016/j.fcl.2012.06.003.

14. Weglein DG, Andersen CR, Morris RP, Buford WL Jr, Panchbhavi VK. Allograft Reconstruction of the Lisfranc Ligament. Foot \& Ankle Specialist 2015;8:292-6. doi:10.1177/1938640015584768.

15. Ahmed S, Bolt B, McBryde A. Comparison of Standard Screw Fixation Versus Suture Button Fixation in Lisfranc Ligament Injuries. Foot Ankle Int 2010;31:892-6. doi:10.3113/ FAI.2010.0892.

16. Jain K, Drampalos E, Clough TM. Results of suture button fixation with targeting device aid for displaced ligamentous Lisfranc injuries in the elite athlete. The Foot 2017;30:43-6. doi:10.1016/j.foot.2017.01.003.
17. Seybold JD, Coetzee JC. Lisfranc Injuries When to Observe, Fix, or Fuse. Clinics in Sports Medicine 2015;34:705-23. doi:10.1016/j.csm.2015.06.006.

18. Weatherford BM, Anderson JG, Bohay DR. Management of Tarsometatarsal Joint Injuries. Journal of the American Academy of Orthopaedic Surgeons 2017;25:469-79. doi:10.5435/JAAOS-D-15-00556.

19. Wang Y, Li Z, Zhang M. Biomechanical study of tarsometatarsal joint fusion using finite element analysis. Medical Engineering and Physics 2014;36:1394-400. doi:10.1016/j. medengphy.2014.03.014.

20. Pelt CE, Bachus KN, Vance RE, Beals TC. A Biomechanical Analysis of a Tensioned Suture Device in the Fixation of the Ligamentous Lisfranc Injury. Foot Ankle Int 2011;32:42231. doi:10.3113/FAI.2011.0422.

21. De los Santos-Real R, Canillas F, Varas-Navas J, MoralesMuñoz P, Barrio-Sanz P, Medina-Santos M. Lisfranc Joint Ligament Complex Reconstruction: A Promising Solution for Missed, Delayed, or Chronic Lisfranc Injury Without Arthritis. The Journal of Foot and Ankle Surgery 2017;56:1350-6. doi:10.1053/j.jfas.2017.06.025.

22. Schneiders W, Schikore H, Rammelt S, Holch J, Heineck J, Zwipp H. Primary open reduction and fixation compared with delayed corrective arthrodesis in the treatment of tarsometatarsal (Lisfranc) fracture dislocation. The Journal of Bone and Joint Surgery-American Volume 2008;90B:1449-506. doi:10.1302/0301-620X.90B11.

23. Brin YS, Nyska M, Kish B. Lisfranc Injury Repair with the TightRope ${ }^{\mathrm{TM}}$ Device: A Short-term Case Series. Foot Ankle Int 2010;31:624-7. doi:10.3113/FAI.2010.0624.

24. Baravarian B, Geffen D. Lisfranc Tightrope. Foot \& Ankle Specialist 2009;2:249-50. doi:10.1177/1938640009347169. 